\title{
Convolutions of heavy tailed random variables and applications to portfolio diversification and MA(1) time series
}

\author{
Jaap Geluk \\ Econometric Institute \\ Erasmus University Rotterdam \\ P.O. Box 1738 \\ 3000 DR Rotterdam, The Netherlands \\ Liang Peng \\ Center for Mathematics and its Applications \\ Australian National University \\ Canberra, ACT 0200, Australia \\ Casper de Vries \\ Erasmus University Rotterdam \\ 3000 DR Rotterdam, The Netherlands
}

October 14, 1999

\begin{abstract}
The paper characterizes first and second order tail behavior of convolutions of i.i.d. heavy tailed random variables with support on the real line. The result is applied to the problem of risk diversification in portfolio analysis and to the estimation of the parameter in a MA(1) model.
\end{abstract}




\section{Introduction}

Assume $X_{1}, X_{2}$ are independent random variables whose distribution function tail sums

$$
\bar{F}_{i}(t):=P\left(\left|X_{i}\right| \geq t\right), i=1,2
$$

are regularly varying with index $-\alpha<0$, i.e.,

$$
\lim _{t \rightarrow \infty} \frac{\bar{F}_{i}(t x)}{\bar{F}_{i}(t)}=x^{-\alpha} \text { for all } x>0 .
$$

For a general overview of regular variation theory the reader is referred to Bingham et al.(1987). The asymptotic behavior of the tail sum of the convolution of $X_{1}$ and $X_{2}$ is studied in Feller (1971). More recently Datta and McCormick (1998) gave the behavior of the tail sum for linear processes $\sum_{i=1}^{\infty} c_{i} X_{t-i}$ under suitable conditions on the coefficients $c_{i}$.

In this paper we investigate the behavior of convolutions in case a refinement of (1.1), called second order regular variation, on the tails of the distribution functions of the random variables holds. Specifically, we assume that the tail sums satisfy

$$
\lim _{t \rightarrow \infty} \frac{\frac{\bar{F}_{i}(t x)}{\bar{F}_{i}(t)}-x^{-\alpha}}{a_{i}(t)}=x^{-\alpha} \frac{x^{\rho}-1}{\rho}
$$

for $x>0, i=1,2$, where $a_{i}$ is a function satisfying $a_{i}(t) \rightarrow 0(t \rightarrow \infty)$. It follows that the functions $\left|a_{i}\right|$ are regularly varying with index $\rho \leq 0$. In case $\rho=0$ read $\frac{x^{\rho}-1}{\rho}=\log x$.

For positive random variables satisfying (1.2) the tail behavior under convolution was studied in Geluk, de Haan, Resnick and Starica (1997) and Geluk and Peng (1999). In this paper we do not make the assumption of positive random variables and provide more precise estimates for a number of cases. We replace the positivity condition with the following tail balance condition

$$
\lim _{t \rightarrow \infty} \frac{\frac{1-F_{i}(t)}{\bar{F}_{i}(t)}-p_{i}}{a_{i}(t)}=r_{i} \in(-\infty, \infty),
$$


where $p_{i} \in[0,1], i=1,2$.

Under the assumptions (1.2) and (1.3) we give an asymptotic expansion for $P\left(X_{1}+X_{2}>t\right)$. It turns out that the number of terms in the expansion depends on the value of the parameter $\alpha$ whereas the type of the terms in the expansion depends on $\alpha, p_{1}, p_{2}, \rho$ and the convergence of the $\alpha$-th moment.

The first application of the main result is on portfolio management. The performance of a portfolio is measured in terms of the returns on investment, i.e. the percentage gain or loss on initial capital. The structure of the portfolio influences the portfolio performance. Some assets have low expected returns and others have high expected returns, but at the cost of higher risk. Perhaps the most important rule of thumb in finance prescribes how one should not structure one's portfolio: 'Don't put all your eggs in one basket'. The idea behind this rule of thumb is that through diversification one can reduce the portfolio risk, measured as the variance of the portfolio return, by virtue of the law of large numbers, since returns on individual assets are imperfectly correlated with each other. The effects of diversification on the mean and variance of a portfolio is well understood and can be found in all elementary textbooks on finance. In this section we elaborate on the virtues of diversification regarding tail risk, about which much less is known.

The tail risk is the probability that there is a very 'large' loss on a portfolio. It depends on the economic context what constitutes a 'large' loss. For a pension fund this constitutes a loss so large that it is unable to pay out the pensions, and for a commercial bank a loss is large if it is unable to meet the cash demand by deposit holders, which may trigger a bank run. It suffices for our purposes to identify the meaning of large by a typical quantile in the left tail of the return distribution. Financial institutions measure the downside risk or tail risk of their proprietary trading portfolio on a daily basis, both for the purpose of internal risk management, and because this is a regulatory requirement (external risk management imposed by public agencies to ensure prudence in the financial sector). This downside risk measurement operation is now commonly known as the Value-at-Risk (VaR) exercise, see e.g. Jorion (1997), Dowd (1998), Danielsson and De Vries $(1997,1998)$ and Longin (1997). Hence it is important to study how the downside risk or VaR is affected by diversification. In section 3 we first give a brief review of first order tail effects and then provide a number of new results on second order refinements based on the result in section 2 . 
Our second application is in time series analysis. Suppose we have observations $Y_{1}, \cdots, Y_{n}$ from the $\mathrm{MA}(1)$ model, i.e.,

$$
Y_{i}=\epsilon_{i}-\theta \epsilon_{i-1}
$$

where $\left\{\epsilon_{i}\right\}$ is a sequence of independent and identically distributed random variables with mean zero and finite variance. In case $|\theta|<1$, the maximum likelihood estimator $\hat{\theta}_{M L E}$ for $\theta$ has the following asymptotic limit:

$$
\sqrt{n}\left(\hat{\theta}_{M L E}-\theta\right) \stackrel{d}{\rightarrow} N\left(0,1-\theta^{2}\right)
$$

(see Brockwell and Davis (1991)). However, in case $|\theta|=1$, the standard asymptotic normal distribution theory does not apply (see Brockwell and Davis (1991)). On the other hand, the normal limit in (1.5) provides a particularly inaccurate approximation for values of $|\theta|$ close to one. For the applications in which inference about $|\theta|$ at or close to one, we refer to Davis, Chen and Dunsmuir (1995) and Davis and Dunsmuir (1996). Moreover, Davis and Dunsmuir (1996) proposed the local maximum estimator $\hat{\theta}_{L M}$, defined as the largest of the local maximizers of the likelihood, and derived the asymptotic limit of $\hat{\theta}_{L M}$ too.

Recently, Davis and Mikosch (1998) obtained the limit behaviour of the local maximizer closest to 1 of the Gaussian likelihood and the corresponding likelihood ratio statistic, used in Davis and Dunsmuir (1996), when $\left\{\epsilon_{i}\right\}$ is an i.i.d. sequence with symmetric stable law with index $\alpha \in(0,2)$. However the limit in Davis and Mikosch (1998) is complicated. For the estimation of $\theta \in(-1,1)$ we refer to Lii and Rosenblatt $(1982,1992)$.

In this paper we give a semi-parametric estimator for $\theta \in[-1,1]$ under the assumption that $\epsilon_{i}$ satisfies

$$
\left\{\begin{array}{l}
P\left(\epsilon_{i}>t\right)=c p t^{-\alpha}(1+o(1)) \\
P\left(\epsilon_{i}<-t\right)=c(1-p) t^{-\alpha}(1+o(1))
\end{array}\right.
$$

as $t \rightarrow \infty$, where $\alpha>0, c>0$ and $p \in[0,1]$. Thus, $\epsilon_{i}$ is in the domain of attraction of a stable law with index $\alpha$ in case $\alpha<2$, and $\epsilon_{i}$ is in the domain of attraction of a normal distribution if $\alpha \geq 2$.

First we give the intuitive derivation of our new estimator. For simplicity we assume to have $n+1$ observations and define $Z_{i}=\left|Y_{i+1}+Y_{i}\right|$ and $W_{i}=$ $\left|Y_{i+1}-Y_{i}\right|(i=1, \cdots, n)$. Let $Z_{n, 1} \leq \cdots \leq Z_{n, n}$ and $W_{n, 1} \leq \cdots \leq W_{n, n}$ denote the order statistics of $Z_{1}, \cdots, Z_{n}$ and $W_{1}, \cdots, W_{n}$, respectively. 
It follows from (1.1), (1.3) and Feller (1971) that as $t \rightarrow \infty$

$$
\begin{aligned}
& P\left(Z_{i}>t\right) \\
= & P\left(Y_{i+1}+Y_{i}>t\right)+P\left(Y_{i+1}+Y_{i}<-t\right) \\
= & \left\{P\left(\epsilon_{i+1}>t\right)+P\left((1-\theta) \epsilon_{i}>t\right)+P\left(-\theta \epsilon_{i-1}>t\right)\right\}(1+o(1)) \\
& +\left\{P\left(\epsilon_{i+1}<-t\right)+P\left((1-\theta) \epsilon_{i}<-t\right)+P\left(-\theta \epsilon_{i-1}<-t\right)\right\}(1+o(1)) \\
= & c\left\{1+(1-\theta)^{\alpha}+|\theta|^{\alpha}\right\} t^{-\alpha}(1+o(1)) .
\end{aligned}
$$

Similarly,

$$
P\left(W_{i}>t\right)=c\left\{1+(1+\theta)^{\alpha}+|\theta|^{\alpha}\right\} t^{-\alpha}(1+o(1)) .
$$

Hence we can estimate $c\left\{1+(1-\theta)^{\alpha}+|\theta|^{\alpha}\right\}$ and $c\left\{1+(1+\theta)^{\alpha}+|\theta|^{\alpha}\right\}$ by $\frac{k}{n} Z_{n, n-k}^{\hat{\alpha}_{Z}(k)}$ and $\frac{m}{n} W_{n, n-m}^{\hat{\alpha}_{W}(m)}$, respectively, where $k=k(n) \rightarrow \infty, k / n \rightarrow 0$, $m=m(n) \rightarrow \infty, m / n \rightarrow 0$ and

$$
\left\{\begin{array}{l}
\hat{\alpha}_{Z}(k):=\left\{\frac{1}{k} \sum_{i=1}^{k} \log Z_{n, n-i+1}-\log Z_{n, n-k}\right\}^{-1} \\
\hat{\alpha}_{W}(m):=\left\{\frac{1}{m} \sum_{i=1}^{m} \log W_{n, n-i+1}-\log W_{n, n-m}\right\}^{-1} .
\end{array}\right.
$$

Note that $\hat{\alpha}_{Z}(k)$ and $\hat{\alpha}_{W}(m)$ are Hill estimators of the tail index $\alpha$ (see Hill (1975)). Since $\hat{\alpha}_{Z}(k)$ and $\hat{\alpha}_{W}(m)$ are consistent estimators of $\alpha$ (see e.g. Mason (1982)), it follows that $\frac{k}{m} Z_{n, n-k}^{\hat{\alpha}_{Z}(k)} W_{n, n-m}^{-\hat{\alpha}_{W}(m)}$ is a consistent estimator of

$$
f(\theta):=\frac{1+|\theta|^{\alpha}+(1-\theta)^{\alpha}}{1+|\theta|^{\alpha}+(1+\theta)^{\alpha}}
$$

Define

$$
f_{n}(\theta):=\frac{1+|\theta|^{\hat{\alpha}_{W}(m)}+(1-\theta)^{\hat{\alpha}_{W}(m)}}{1+|\theta|^{\hat{\alpha}_{W}(m)}+(1+\theta)^{\hat{\alpha}_{W}(m)}} .
$$

It is easy to check that $f_{n}(\theta)$ is a decreasing function of $\theta$ on the interval $[-1,1]$. Let $f_{n}^{-}(\theta)$ denote the inverse function of $f_{n}(\theta)$. It follows that

$$
\hat{\theta}_{n}:=f_{n}^{-}\left(\frac{k}{m} Z_{n, n-k}^{\hat{\alpha}_{Z}(k)} W_{n, n-m}^{-\hat{\alpha}_{W}(m)}\right)
$$

is a consistent estimator of $\theta$.

In order to prove asymptotic normality of $\hat{\theta}_{n}$, we need second order regular variation conditions for both $P\left(Z_{i}>t\right)$ and $P\left(W_{i}>t\right)$. In section 4 these conditions are obtained from assumptions on the innovations using theorem 2.1 . 


\section{Second order behaviour of convolutions}

Theorem 2.1. Let $X_{i}, i=1,2$ be independent random variables with distribution functions $F_{i}$ satisfying (1.2) and (1.3). Define for $j \geq 0$ integer and $\alpha>0$,

$$
c_{\alpha, j}=\frac{\Gamma(\alpha+j)}{j ! \Gamma(\alpha)}
$$

Consider the following cases

A. If $0<\alpha<1$ then as $t \rightarrow \infty$

$$
\begin{array}{r}
P\left(X_{1}+X_{2}>t\right)=\sum_{i=1}^{2}\left(r_{i}+o(1)\right) \bar{F}_{i}(t) a_{i}(t) \\
+\sum_{i=1}^{2} p_{i} \bar{F}_{i}(t)+(d+o(1)) \bar{F}_{1}(t) \bar{F}_{2}(t)
\end{array}
$$

where

$$
d=p_{1} p_{2}\left\{-\frac{\Gamma(1-\alpha)^{2}}{\Gamma(1-2 \alpha)}+\frac{2 \Gamma(1-\alpha) \Gamma(2 \alpha)}{\Gamma(\alpha)}\right\}-\left(p_{1}+p_{2}\right) \frac{\Gamma(1-\alpha) \Gamma(2 \alpha)}{\Gamma(\alpha)} .
$$

B. If $\alpha \geq 1$ and $E\left|X_{i}\right|^{\alpha}<\infty(i=1,2)$, then as $t \rightarrow \infty$

$$
\begin{aligned}
P\left(X_{1}\right. & \left.+X_{2}>t\right)=\sum_{i=1}^{2}\left(r_{i}+o(1)\right) \bar{F}_{i}(t) a_{i}(t) \\
& +\sum_{i=1}^{2} p_{i} \bar{F}_{i}(t)\left\{\sum_{j=0}^{[\alpha]-1} c_{\alpha, j} \frac{E X_{3-i}^{j}}{t^{j}}+\left(c_{\alpha,[\alpha]}+o(1)\right) \frac{E X_{3-i}^{[\alpha]}}{t^{[\alpha]}}\right\},
\end{aligned}
$$

where $[\alpha]$ is the greatest integer less than or equal $\alpha$.

C. If $E\left|X_{i}\right|^{\alpha}=\infty(i=1,2)$ and one of the following holds:

1. $\alpha \geq 1$ is even 
2. $\alpha \geq 1$ is odd and $p_{i} \neq \frac{1}{2}(i=1,2)$

3. $\alpha \geq 1$ is odd, $p_{i}=\frac{1}{2}, \rho=0$ and $r_{i} \neq 0(i=1,2)$

4. $\alpha \geq 1$ is odd, $\rho<0$ and $r_{i} \neq 0(i=1,2)$,

then as $t \rightarrow \infty$

$$
\begin{aligned}
& P\left(X_{1}+X_{2}>t\right)= \\
& =\sum_{i=1}^{2}\left(r_{i}+o(1)\right) \bar{F}_{i}(t) a_{i}(t)+\sum_{i=1}^{2} p_{i} \bar{F}_{i}(t)\left\{\sum_{j=0}^{\alpha-1} c_{\alpha, j} \frac{E X_{3-i}^{j}}{t^{j}}+\right. \\
& \left.+\left(\alpha c_{\alpha, \alpha}+o(1)\right) \frac{1}{t^{\alpha}} \int_{0}^{t}\left(1-F_{3-i}(y)+(-1)^{\alpha} F_{3-i}(-y)\right) y^{\alpha-1} d y\right\} .
\end{aligned}
$$

D. If $E\left|X_{i}\right|^{\alpha}=\infty(i=1,2)$ and $\alpha>1$ is non-integer, then as $t \rightarrow \infty$

$$
\begin{aligned}
P\left(X_{1}\right. & \left.+X_{2}>t\right)=\sum_{i=1}^{2}\left(r_{i}+o(1)\right) \bar{F}_{i}(t) a_{i}(t)+ \\
& +\sum_{i=1}^{2} p_{i} \bar{F}_{i}(t) \sum_{j=0}^{[\alpha]-1} c_{\alpha, j} \frac{E X_{3-i}^{j}}{t^{j}}+\left(h_{\alpha}+o(1)\right) \bar{F}_{1}(t) \bar{F}_{2}(t),
\end{aligned}
$$

where $[\alpha]$ is the greatest integer less than or equal $\alpha$ and $h_{\alpha}$ is a constant.

The following result (see de Haan and Pereira (1999)) is needed for the proof of the theorem.

Lemma 2.1. Let $f$ be a measurable function and for some function $a_{1}(t)>0$ we have

$$
\lim _{t \rightarrow \infty} \frac{f(t x)-f(t)}{a_{1}(t)}=\frac{x^{\gamma}-1}{\gamma}
$$

for all $x>0$ where $\gamma$ is a real parameter. Then there exists a positive function a with $a(t) \sim a_{1}(t)(t \rightarrow \infty)$ with the property that for every $\varepsilon, \varepsilon^{\prime}>0$ there exists a $t_{0}>0$ such that for $t \geq t_{0}, t x \geq t_{0}$

$$
x^{-\gamma} e^{-\varepsilon^{\prime}|\log x|}\left|\frac{f(t x)-f(t)}{a(t)}-\frac{x^{\gamma}-1}{\gamma}\right|<\varepsilon .
$$


Proof of Theorem 2.1. Note that

$$
\begin{aligned}
& P\left(X_{1}+X_{2}>t\right)-P\left(X_{1}>\frac{t}{2}\right) P\left(X_{2}>\frac{t}{2}\right)= \\
& =\sum_{i=1}^{2} \int_{-\infty}^{t / 2} P\left(X_{i}>t-y\right) d F_{3-i}(y) \\
& =\sum_{i=1}^{2} \bar{F}_{i}(t) a_{i}(t) \int_{-\infty}^{t / 2} \frac{\frac{P\left(X_{i}>t-y\right)}{\bar{F}_{i}(t)}-p_{i}\left(1-\frac{y}{t}\right)^{-\alpha}}{a_{i}(t)} d F_{3-i}(y) \\
& \quad+\sum_{i=1}^{2} p_{i} \bar{F}_{i}(t) \int_{-\infty}^{t / 2}\left(1-\frac{y}{t}\right)^{-\alpha} d F_{3-i}(y) .
\end{aligned}
$$

Denote the integrals on the right hand side with $I_{i}$ and $J_{i}$ respectively.

Substituting the balance condition $P\left(X_{i}>t-y\right)=p_{i} \bar{F}_{i}(t-y)+\left(r_{i}+\right.$ $o(1)) \bar{F}_{i}(t-y) a_{i}(t-y)$ as $t \rightarrow \infty$ (valid uniformly for $y \in(-\infty, t / 2)$ ) we have

$$
\begin{aligned}
I_{i} & =p_{i} \int_{-\infty}^{t / 2} \frac{\frac{\bar{F}_{i}(t-y)}{\bar{F}_{i}(t)}-\left(1-\frac{y}{t}\right)^{-\alpha}}{a_{i}(t)} d F_{3-i}(y)+ \\
& +\left(r_{i}+o(1)\right) \int_{-\infty}^{t / 2} \frac{\bar{F}_{i}(t-y)}{\bar{F}_{i}(t)} \frac{a_{i}(t-y)}{a_{i}(t)} d F_{3-i}(y)=: I_{i 1}+I_{i 2}
\end{aligned}
$$

Now $I_{i 1} \rightarrow 0$ as $t \rightarrow \infty$ follows using dominated convergence. The dominating function is provided with the above Lemma. Similarly Potter's inequality (see e.g. Bingham et al.(1987)) gives the dominating function which is necessary to apply dominated convergence in $I_{i 2}$. This gives $I_{i 2} \rightarrow r_{i}$ as $t \rightarrow \infty$.

The method of estimation of the integral $J_{i}$ depends on the value of $\alpha$.

A. Suppose $\alpha<1$. Integration by parts gives

$$
\begin{aligned}
\int_{0}^{t / 2}\{ & \left.\left(1-\frac{y}{t}\right)^{-\alpha}-1\right\} d F_{i}(y)= \\
& =-\left(2^{\alpha}-1\right) P\left(X_{i}>\frac{t}{2}\right)+\alpha \int_{0}^{1 / 2} P\left(X_{i}>t y\right)(1-y)^{-\alpha-1} d y .
\end{aligned}
$$

Using $P\left(X_{i}>\frac{t}{2}\right) \sim p_{i} \bar{F}_{i}\left(\frac{t}{2}\right) \sim p_{i} 2^{\alpha} \bar{F}_{i}(t)$ and regular variation of $\bar{F}_{i}$ we 
find

$$
\begin{aligned}
& \int_{0}^{t / 2}\left\{\left(1-\frac{y}{t}\right)^{-\alpha}-1\right\} d F_{i}(y) \\
& \sim p_{i}\left\{-\left(2^{\alpha}-1\right) 2^{\alpha}+\alpha \int_{0}^{\frac{1}{2}} y^{-\alpha}(1-y)^{-\alpha-1} d y\right\} \bar{F}_{i}(t)
\end{aligned}
$$

Another integration by parts gives

$$
\begin{aligned}
& \int_{-\infty}^{0}\left\{\left(1-\frac{y}{t}\right)^{-\alpha}-1\right\} d F_{i}(y) \\
& =-\alpha \int_{0}^{\infty} F_{i}(-t y)(1+y)^{-\alpha-1} d y
\end{aligned}
$$

hence

$$
\begin{aligned}
& \int_{-\infty}^{0}\left\{\left(1-\frac{y}{t}\right)^{-\alpha}-1\right\} d F_{i}(y) \\
& \sim \quad-\alpha\left(1-p_{i}\right) \bar{F}_{i}(t) \int_{0}^{\infty} y^{-\alpha}(1+y)^{-\alpha-1} d y
\end{aligned}
$$

Combination of the estimates now gives

$$
\begin{aligned}
J_{i}= & F_{3-i}\left(\frac{t}{2}\right)+\left\{-\left(2^{\alpha}-1\right) 2^{\alpha} p_{3-i}+\alpha p_{3-i} \int_{0}^{\frac{1}{2}} y^{-\alpha}(1-y)^{-\alpha-1} d y\right. \\
& \left.-\alpha\left(1-p_{3-i}\right) \int_{0}^{\infty} y^{-\alpha}(1+y)^{-\alpha-1} d y+o(1)\right\} \bar{F}_{3-i}(t) \\
= & 1+\left\{-p_{3-i} 2^{2 \alpha}+\alpha p_{3-i} \int_{0}^{\frac{1}{2}} y^{-\alpha}(1-y)^{-\alpha-1} d y\right. \\
& \left.-\alpha\left(1-p_{3-i}\right) \int_{0}^{\infty} y^{-\alpha}(1+y)^{-\alpha-1} d y+o(1)\right\} \bar{F}_{3-i}(t) .
\end{aligned}
$$

Note that

$$
\prod_{i=1}^{2} P\left(X_{i}>\frac{t}{2}\right) \sim \prod_{i=1}^{2} p_{i} 2^{\alpha} \bar{F}_{i}(t) .
$$

Substitution of (2.4), (2.5) and (2.6) in (2.3) gives (2.2), where

$$
\begin{aligned}
d= & p_{1} p_{2}\left\{-2^{2 \alpha}+2 \alpha \int_{0}^{\frac{1}{2}} y^{-\alpha}(1-y)^{-\alpha-1} d y+2 \alpha \int_{0}^{\infty} y^{-\alpha}(1+y)^{-\alpha-1} d y\right\} \\
& -\alpha\left(p_{1}+p_{2}\right) \int_{0}^{\infty} y^{-\alpha}(1+y)^{-\alpha-1} d y
\end{aligned}
$$


The stated representation now follows since

$$
2 \alpha \int_{0}^{\frac{1}{2}} y^{-\alpha}(1-y)^{-\alpha-1} d y=2^{2 \alpha}-\frac{\Gamma(1-\alpha)^{2}}{\Gamma(1-2 \alpha)}
$$

and

$$
\int_{0}^{\infty} y^{-\alpha}(1+y)^{-\alpha-1} d y=\frac{\Gamma(1-\alpha) \Gamma(2 \alpha)}{\Gamma(1+\alpha)}
$$

B. Suppose $\alpha \geq 1$ and $E\left|X_{i}\right|^{\alpha}<\infty(i=1,2)$. Since

$$
\frac{(1+x)^{-\alpha}-\sum_{j=0}^{[\alpha]-1}\left(\begin{array}{c}
-\alpha \\
j
\end{array}\right) x^{j}}{x^{[\alpha]}} \rightarrow \frac{(-\alpha)(-\alpha-1) \ldots(-\alpha-[\alpha]+1)}{[\alpha] !}, \quad(x \rightarrow 0)
$$

the above ratio is bounded for $x \in\left(-\infty, \frac{1}{2}\right) .([\alpha]$ is the greatest integer less than or equal to $\alpha$.) Hence we may use dominated convergence in order to find

$$
\begin{aligned}
\int_{-\infty}^{t / 2} y^{[\alpha]} & \frac{\left(1-\frac{y}{t}\right)^{-\alpha}-\sum_{j=0}^{[\alpha]-1}\left(\begin{array}{c}
-\alpha \\
j
\end{array}\right)\left(-\frac{y}{t}\right)^{j}}{\left(-\frac{y}{t}\right)[\alpha]} d F_{3-i}(y) \rightarrow \\
& \rightarrow \frac{(-\alpha)(-\alpha-1) \ldots(-\alpha-[\alpha]+1)}{[\alpha] !} \int_{-\infty}^{\infty} y^{[\alpha]} d F_{3-i}(y)
\end{aligned}
$$

as $t \rightarrow \infty$.

Since $\overline{F_{i}}$ is regularly varying, we have for $j=0, \ldots,[\alpha]-1$

$\int_{t / 2}^{\infty} y^{j} d F_{i}(y)=O\left(t^{j} \bar{F}_{i}(t)\right)$ by Karamata's theorem (see e.g. Bingham et al.(1987)). Combination of this observation with (2.7) shows that for the case under consideration we have as $t \rightarrow \infty$

$$
J_{i}=\sum_{j=0}^{[\alpha]-1} c_{\alpha, j} \frac{E X_{3-i}^{j}}{t^{j}}+\left(c_{\alpha,[\alpha]}+o(1)\right) \frac{E X_{3-i}^{[\alpha]}}{t^{[\alpha]}}+O\left(\bar{F}_{3-i}(t)\right),
$$

where $c_{\alpha, j}$ is as in (2.1).

Substituting (2.7) and (2.8) in (2.3) gives

$$
\begin{aligned}
& P\left(X_{1}+X_{2}>t\right)=P\left(X_{1}>\frac{t}{2}\right) P\left(X_{2}>\frac{t}{2}\right)+\sum_{i=1}^{2}\left(r_{i}+o(1)\right) \bar{F}_{i}(t) a_{i}(t)+ \\
& +\sum_{i=1}^{2} p_{i} \bar{F}_{i}(t)\left\{\sum_{j=0}^{[\alpha]-1} c_{\alpha, j} \frac{E X_{3-i}^{j}}{t^{j}}+\left(c_{\alpha,[\alpha]}+o(1)\right) \frac{E X_{3-i}^{[\alpha]}}{t^{[\alpha]}}+O\left(\bar{F}_{3-i}(t)\right)\right\} .
\end{aligned}
$$


The result follows since $E\left|X_{i}\right|^{[\alpha]}<\infty$ implies

$$
t^{[\alpha]} \bar{F}_{i}(t) \rightarrow 0 \text { as } t \rightarrow \infty
$$

hence $\prod_{i=1}^{2} P\left(X_{i}>\frac{t}{2}\right)=O\left(\prod_{i=1}^{2} \bar{F}_{i}(t)\right)=o\left(t^{-[\alpha]} \wedge_{i=1}^{2} \bar{F}_{i}(t)\right)$.

C1,C2 Suppose $E\left|X_{i}\right|^{\alpha}=\infty(i=1,2), \alpha$ is integer and one of the following holds: $1 . \alpha \geq 1$ is even $2 . \alpha \geq 1$ is odd and $p_{i} \neq \frac{1}{2}$.

Write $J_{i}=\int_{-\infty}^{t / 2}\left(1-\frac{y}{t}\right)^{-\alpha} d F_{3-i}(y)=: J_{1 i}+J_{2 i}$, where $J_{1 i}$ and $J_{2 i}$ are the integrals over $\left(-\infty,-\frac{t}{2}\right)$ and $\left(-\frac{t}{2}, \frac{t}{2}\right)$ respectively. Then $\left|J_{1 i}\right| \leq 2^{\alpha} F_{3-i}\left(-\frac{t}{2}\right) \leq$ $2^{\alpha} \bar{F}_{3-i}\left(\frac{t}{2}\right)=O\left(\bar{F}_{3-i}(t)\right)$, using regular variation of $\bar{F}_{3-i}$. In order to estimate $\left|J_{2 i}\right|$ note that for $|y| \leq t / 2$

$$
\left(1-\frac{y}{t}\right)^{-\alpha}=1+\sum_{j=1}^{\alpha} c_{\alpha, j}\left(\frac{y}{t}\right)^{j}+c_{\alpha, \alpha+1}\left(\frac{\theta y}{t}\right)^{\alpha+1},
$$

where $|\theta|=|\theta(y, t)| \leq 1$.

For $j=0, \ldots, \alpha-1$ Karamata's theorem shows that as $t \rightarrow \infty$

$$
\frac{1}{t^{j}} \int_{-t / 2}^{t / 2} y^{j} d F_{3-i}(y)=\frac{E X_{3-i}^{j}}{t^{j}}+O\left(\bar{F}_{3-i}(t)\right) .
$$

Integration by parts gives

$$
\begin{aligned}
& \frac{1}{t^{\alpha}} \int_{-t / 2}^{t / 2} y^{\alpha} d F_{3-i}(y)=-\frac{1}{2^{\alpha}}\left(1-F_{3-i}\left(\frac{t}{2}\right)+(-1)^{\alpha} F_{3-i}\left(-\frac{t}{2}\right)\right) \\
& +\frac{\alpha}{t^{\alpha}} \int_{0}^{t / 2}\left(1-F_{3-i}(y)+(-1)^{\alpha} F_{3-i}(-y)\right) y^{\alpha-1} d y \\
& \sim \frac{\alpha}{t^{\alpha}} \int_{0}^{t}\left(1-F_{3-i}(y)+(-1)^{\alpha} F_{3-i}(-y)\right) y^{\alpha-1} d y=: K_{i}(t)
\end{aligned}
$$

where the asymptotic equality follows by Karamata's theorem. In case $j=\alpha+1$ we have similarly

$$
\frac{1}{t^{\alpha+1}} \int_{-t / 2}^{t / 2} y^{\alpha+1} d F_{3-i}(y)=O\left(\bar{F}_{3-i}(t)\right) .
$$

Collecting the above estimates it follows that 


$$
\begin{aligned}
J_{i}= & F_{3-i}\left(\frac{t}{2}\right)-F_{3-i}\left(-\frac{t}{2}\right)+O\left(\bar{F}_{3-i}(t)\right)+\sum_{j=1}^{\alpha-1} c_{\alpha, j} \frac{E X_{3-i}^{j}}{t^{j}} \\
& +\left(c_{\alpha, \alpha}+o(1)\right) \frac{\alpha}{t^{\alpha}} \int_{0}^{t}\left(1-F_{3-i}(y)+(-1)^{\alpha} F_{3-i}(-y)\right) y^{\alpha-1} d y \\
=1 & +\sum_{j=1}^{\alpha-1} c_{\alpha, j} \frac{E X_{3-i}^{j}}{t^{j}}+ \\
& +\left(c_{\alpha, \alpha}+o(1)\right) \frac{\alpha}{t^{\alpha}} \int_{0}^{t}\left(1-F_{3-i}(y)+(-1)^{\alpha} F_{3-i}(-y)\right) y^{\alpha-1} d y .
\end{aligned}
$$

Note that since $\bar{F}_{3-i}$ is regularly varying with index $-\alpha$, we have $\bar{F}_{3-i}(t)=$ $o\left(K_{i}(t)\right)$, hence as $t \rightarrow \infty \prod_{i=1}^{2} P\left(X_{i}>t / 2\right)=o\left(\sum_{i=1}^{2} K_{i}(t) \bar{F}_{i}(t)\right)$.

The result now follows if we combine the estimates for $I_{i}$ and $J_{i}$.

C3, C4 Suppose $\alpha$ odd, $p_{i}=\frac{1}{2}, r_{i} \neq 0$ for $i=1,2$. Using (1.3) it follows that $1-F_{i}(t)+(-1)^{\alpha} F_{i}(-t) \sim 2 r_{i} a_{i}(t) F_{i}(t)$, hence this function is (in absolute value) regularly varying with index $\rho-\alpha$. It follows that, if $\rho=0,(2.12)$ holds again and we have the same estimate as in C1 and C2.

The case $\mathrm{C} 4$ is similar.

D Suppose $E\left|X_{i}\right|^{\alpha}=\infty(i=1,2)$ and $\alpha>1$ non-integer

In this case we need a more precise estimate for $J_{i}$. An integration by parts shows that

$$
\begin{aligned}
J_{1 i} & =\int_{-\infty}^{-t / 2}\left(1-\frac{y}{t}\right)^{-\alpha} d F_{3-i}(y) \\
& =\left(\frac{3}{2}\right)^{-\alpha} F_{3-i}\left(-\frac{t}{2}\right)-\alpha \int_{-\infty}^{-1 / 2} F_{3-i}(t y)(1-y)^{-\alpha-1} d y \\
& \sim(1-p)\left[\left(\frac{3}{2}\right)^{-\alpha} \bar{F}_{3-i}\left(\frac{t}{2}\right)-\alpha \int_{-\infty}^{-1 / 2} \bar{F}_{3-i}(-t y)(1-y)^{-\alpha-1} d y\right] \\
& \sim(1-p) \bar{F}_{3-i}(t)\left[\left(\frac{4}{3}\right)^{\alpha}-\alpha \int_{-\infty}^{-1 / 2}(-y)^{-\alpha}(1-y)^{-\alpha-1} d y\right] .
\end{aligned}
$$

Now (2.11) is replaced with the estimate (valid for $j=0, \ldots,[\alpha]$ )

$$
\frac{1}{t^{j}} \int_{-t / 2}^{t / 2} y^{j} d F_{3-i}(y)=\frac{E X_{3-i}^{j}}{t^{j}}+\frac{\alpha}{\alpha-j} \bar{F}_{3-i}(t)+o\left(\bar{F}_{3-i}(t)\right) .
$$


In case $j=[\alpha]+1$ we have as $\rightarrow \infty$

$$
\frac{1}{t^{[\alpha]+1}} \int_{-t / 2}^{t / 2} y^{[\alpha]+1} d F_{3-i}(y) \sim \frac{\alpha}{[\alpha]+1-\alpha} 2^{\alpha-[\alpha]-1} \bar{F}_{3-i}(t) .
$$

The rest of the proof follows as before.

\section{Portfolio diversification effects}

\subsection{First order effects}

It is a stylized fact that financial asset returns are heavy tailed distributed as in (1.1). Then Feller's (1971, VIII.8) classical result can be used to investigate the benefits from cross-sectional portfolio diversification.

To this end we first briefly have to review the standard finance model for (relative) risk pricing. In the so called Capital Asset Pricing Model (CAPM), see e.g. Fama and Miller (1972) and Copeland and Weston (1983, ch.7), the return $R_{i}$ on an individual asset $i$ is related to the return $r_{f}$ on the riskfree asset (government bond) and the return $R$ on the market portfolio (measured as the return on an index like the S\&P500). Suppose that

$$
R_{i}-r_{f}=\beta_{i}\left(R-r_{f}\right)+Q_{i},
$$

where $Q_{i}$ is the idiosyncratic or unsystematic risk factor of the return $R_{i}$ on asset $i$, and $\beta_{i}$ is the correlation coefficient in a regression of $R_{i}-r_{f}$ on $R-r_{f}$. The typical assumption is that $Q_{i}, Q_{j}$ and $R$ are cross-sectionally independently distributed. Thus $\beta_{i}$ reflects how $R_{i}$ covaries with the market. The CAPM holds that market forces determine what happens in expectation

$$
E\left[R_{i}-r_{f}\right]=\beta_{i} E\left[R-r_{f}\right] .
$$

Since for what follows the risk free rate $r_{f}$ plays no role, we economize on notation and set $r_{f}=0$.

Consider a portfolio of $m$ assets with weights $w_{i}, w_{i}>0, \sum_{1}^{m} w_{i}=1$. We focus on equally weighted portfolios $w_{i}=1 / m$. Let $\bar{\beta}=\frac{1}{m} \sum_{1}^{m} \beta_{i}$. Dacorogna et al. (1998) report the following diversification result:

Lemma 3.1 (diversification benefits). Suppose the $Q_{i}$ are cross sectionally i.i.d. distributed and satisfy (1.1). For large loss levels the conditional 
tail diversification benefits from the equally weighted portfolio are larger if the returns have finite variance but are heavy tailed, than if they are normally distributed. Specifically we find as $x \rightarrow-\infty$ that

$$
P\left\{\frac{1}{m} \sum_{1}^{m} R_{i}<x \mid R=r\right\} \sim P\left\{\frac{1}{m^{1-1 / \alpha}} Q_{i}+\bar{\beta} R<x \mid R=r\right\} .
$$

Diversification against tail risk is more effective if returns are heavy tailed distributed with $\alpha>2$ than if the underlying distribution is normal. Recall that under normality risk is reduced by the square root of $m$. It has been noted in the economics literature that the effect of diversification is less pronounced if $\alpha<2$ in comparison with the normal distribution. Fama and Miller (1972, p. 270) discuss the case of sum stable distributions. They note that for $\alpha<1$ diversification actually increases the dispersion, and hence putting all eggs in the same basket is advisable in such cases. But financial data do not display such heavy tails, rather $\alpha>2$. We are not aware of a discussion of downside risk diversification in case $\alpha>2$.

Note that the above result is conditional. The motivation for stating it this way is that through diversification one can reduce the contribution of the unsystematic risk factors $Q_{i}$ to the total risk, but one cannot get rid of the contribution of the systematic risk factor $R$. Nevertheless the following is straightforward.

Lemma 3.2. Suppose the $R$ and the $Q_{i}$ are i.i.d. distributed and satisfy (1.1) with the same scale coefficient and tail index. Then

$$
P\left\{\frac{1}{m} \sum_{1}^{m} R_{i}<x\right\} \sim P\left\{\left(m^{1-\alpha}+(\bar{\beta})^{\alpha}\right)^{1 / \alpha} Q_{i}<x\right\} \text { as } x \rightarrow-\infty .
$$

\subsection{Second order diversification benefits}

Since the asset returns can be positive or negative, we need a result on convolutions with heavy tails on both sides. Under continuous compounding the whole real line is the support of the return distribution for assets like equity and foreign exchange. Assume furthermore that the tail index $\alpha>$ 2 for both tails. Financial data usually indicate that the mean and the variance are finite. We specialize the general result of the paper to two special cases which are of interest from an economic point of view. To restrict the

number of different combinations that will arise, we assume that the tails are 
symmetric. This is a reasonable assumption for e.g. foreign currency return data when the exchange rate is left freely floating. Other possibilities are left to the reader.

Corollary 1 (Similar tail behavior). Suppose that as $x \rightarrow \infty$,

$$
\begin{aligned}
& P\{X>x\}=a x^{-\alpha}\left(1+b x^{\rho}+o\left(x^{\rho}\right)\right) \quad(a>0, b \neq 0), \\
& P\{X \leq-x\}=a x^{-\alpha}\left(1+b x^{\rho}+o\left(x^{\rho}\right)\right) \quad(a>0, b \neq 0) \text {. }
\end{aligned}
$$

Moreover, assume that $\alpha>2, \rho<0$, so that $E[X]$ and $E\left[X^{2}\right]$ are finite. Suppose $X_{1}$ and $X_{2}$ are i.i.d. and satisfy (3.2). Then application of theorem 2.1 gives

$$
\begin{aligned}
P\{ & \left.X_{1}+X_{2}>x\right\}=P\left\{X_{1}+X_{2} \leq-x\right\} \\
= & \left.2 a x^{-\alpha}\left(1+b x^{\rho}+\alpha E[X] x^{-1}+\frac{\alpha(\alpha+1)}{2} E\left[X^{2}\right] x^{-2}\right)\right)+ \\
& +o\left(x^{-\alpha-2}\right)+o\left(x^{-\alpha+\rho}\right)
\end{aligned}
$$

as $x \rightarrow \infty$.

We find that because the distribution of asset returns is two-sided, vis a vis the case of positive random variables considered in Geluk et al. (1997), a new factor depending on $E\left[X^{2}\right]$ enters as the second order term if $E[X]=0$ and $\rho \leq-2$. Again, for the case of freely floating exchange rates one typically finds that the mean is zero. But for other assets like equity a positive mean is more reasonable case since the mean equity returns reflect the positive long run growth rate of the economy. For the purpose of diversification we iterate further and find:

Corollary 2. Under the conditions of Corollary 1 if $\rho<-1$ and $E[X]>0$ we have as $x \rightarrow \infty$

$$
P\left\{\frac{1}{m} \sum_{1}^{m} X_{i} \leq-x\right\}=m^{1-\alpha} a s^{-\alpha}\left(1+\frac{m-1}{m} \alpha E[X] x^{-1}+o\left(x^{-1}\right)\right) ;
$$

while if $E[X]=0$ and $\rho<-2$

$P\left\{\frac{1}{m} \sum_{1}^{m} X_{i} \leq-x\right\}=m^{1-\alpha} a s^{-\alpha}\left(1+\frac{m-1}{m^{2}} \frac{\alpha(\alpha+1)}{2} E\left[X^{2}\right] x^{-2}+o\left(x^{-2}\right)\right)$. 
We return to the question of diversification. A more precise evaluation of the diversification benefits for equally weighted portfolios is given in the next result.

Proposition 1. Under the conditions of Lemma 3.1 and Corollary 2, for the case of equation (3.6) and when $m>1$

$$
\begin{aligned}
P\left\{\frac{1}{m} \sum_{1}^{m} R_{i}\right. & \leq-x \mid R=r\} \\
& =m^{1-\alpha} a x^{-\alpha}\left(1+\left\{\frac{m-1}{m} \alpha E\left[Q_{i}\right]+\alpha \bar{\beta} r\right\} x^{-1}+o\left(x^{-1}\right)\right)
\end{aligned}
$$

while if equation (3.7) applies we get for $m>1$

$$
\begin{gathered}
P\left\{\frac{1}{m} \sum_{1}^{m} R_{i} \leq-x \mid R=r\right\} \\
=m^{1-\alpha} a x^{-\alpha}\left(1+\alpha \bar{\beta} r x^{-1}+\frac{\alpha(\alpha+1)}{2}\left\{\frac{m-1}{m^{2}} E\left[Q_{i}^{2}\right]+(\bar{\beta} r)^{2}\right\} x^{-2}+o\left(x^{-2}\right)\right) .
\end{gathered}
$$

Proof. Combine Lemma 3.1 and Corollary 2 repeatedly. Finally calculate the shift due to the translation of $\frac{1}{m} \sum_{1}^{m} Q_{i}$ by $\bar{\beta} r$.

Remark 1. If $R$ also satisfies (3.2) but with a first order tail index $\alpha_{R}>$ $\alpha_{Q}+2$, or if the cdf of $R$ has light tails, then we can replace $r$ in the Proposition (1) by the expectation $E[R]$.

Remark 2. If $\rho=-1$ then (3.8) becomes

$$
\begin{aligned}
P\left\{\frac{1}{m} \sum_{1}^{m} R_{i} \leq\right. & -x \mid R=r\}= \\
& m^{1-\alpha} a x^{-\alpha}\left(1+\left(\frac{m-1}{m} \alpha E\left[Q_{i}\right]+\alpha \bar{\beta} r+\frac{b}{m}\right) x^{-1}+o\left(x^{-1}\right)\right) ;
\end{aligned}
$$

while if $\rho=-2$ then (3.9) becomes

$$
\begin{gathered}
P\left\{\frac{1}{m} \sum_{1}^{m} R_{i} \leq-x \mid R=r\right\} \\
=m^{1-\alpha} a x^{-\alpha}\left(1+\alpha \bar{\beta} r x^{-1}+\left\{\frac{\alpha(\alpha+1)}{2}\left(\frac{m-1}{m^{2}} E\left[Q_{i}^{2}\right]+(\bar{\beta} r)^{2}\right)+\frac{b}{m^{2}}\right\} x^{-2}+o\left(x^{-2}\right)\right) .
\end{gathered}
$$


Example 1 (Student). An example may help to clarify what the propositions actually imply. Suppose we can take an open position in one or two currencies and suppose that the interest rates across the three countries are equal. In that case investing abroad is just a fair gamble. Furthermore assume that the two exchange rates are i.i.d. Empirically the Student-t with say 3 degrees of freedom is known to give a decent fit to foreign currency return data. The density reads

$$
f(x)=2 \pi^{-1} 3^{-1 / 2}\left[1+x^{2} / 3\right]^{-2} .
$$

It follows that (3.2) holds with $\alpha=3, \rho=-2, a=2 \sqrt{3} / \pi, b=-18 / 5$. From (3.10) we compute the effect of diversification. Putting all money in a single currency gives downside risk equal to

$$
P\{X \leq-x\}=\frac{2 \sqrt{3}}{\pi} x^{-3}\left(1-\frac{18}{5} x^{-2}+o\left(x^{-2}\right)\right) .
$$

Application of theorem 2.1 shows that since $E[X]=0$ and $\rho=-2$, the second order term consists of two parts

$$
\left(b+\frac{\alpha(\alpha+1)}{2} E\left[X^{2}\right]\right) x^{-2}=\left(b+\frac{1}{2} \frac{\alpha^{2}(\alpha+1)}{\alpha-2}\right) x^{-2}=\left(-\frac{18}{5}+\frac{36}{2}\right) x^{-2} .
$$

Hence diversification by buying equal shares into the two currencies gives downside risk equal to

$$
\begin{aligned}
P\left\{\frac{X_{1}+X_{2}}{2}\right. & \leq-x\} \\
& =2^{1-3} \frac{2 \sqrt{3}}{\pi} x^{-3}\left(1-\frac{18}{5} 2^{-2} x^{-2}+\frac{3(3+1)}{2} E\left[X^{2}\right] 2^{-2} x^{-2}+o\left(x^{-2}\right)\right) \\
& =\frac{1}{4} \frac{2 \sqrt{3}}{\pi} x^{-3}\left(1+\frac{18}{5} x^{-2}+o\left(x^{-2}\right)\right) .
\end{aligned}
$$

Since the first order scale coefficient of the diversified portfolio is only one-fourth of the first order scale coefficient of the undiversified portfolio, diversification is an important help for reducing the tail risk. Nevertheless, due to the switch in sign of the second order scale coefficient, diversification does not always reduce the tail risk. Let $Y=7^{1 / 3} X_{2}$. Hence

$$
P\{Y \leq-x\}=7 \frac{2 \sqrt{3}}{\pi} x^{-3}\left(1-7^{2 / 3} \frac{18}{5} x^{-2}+o\left(x^{-2}\right)\right)
$$


For the diversified portfolio consisting of $X_{1}$ and $Y$, the tail risk is

$$
P\left\{\frac{X_{1}+Y}{2} \leq-x\right\}=\frac{2 \sqrt{3}}{\pi} x^{-3}\left(1+\frac{153-9.7^{2 / 3}}{40} x^{-2}+o\left(x^{-2}\right)\right) .
$$

It follows that for large threshold levels $x$ one is better off by putting all money in $X_{1}$, rather than to diversify and put halve of the investment into $Y$. The reason is that while the portfolio $\frac{X_{1}+Y}{2}$ and $X_{1}$ have identical first order coefficients $\frac{2 \sqrt{3}}{\pi} x^{-3}$, the second order scale coefficient of the portfolio $\frac{X_{1}+Y}{2}$ is positive and adds to the tail risk, and the opposite holds for the second order scale coefficient of $X_{1}$. The example thus shows the relevance of the second order terms for portfolio selection problems.

\section{Asymptotic normality of $\hat{\theta}_{n}$}

In order to obtain the limiting behavior of $\hat{\theta}_{n}$, we need a stricter condition than (1.1). Assume as $t \rightarrow \infty$

$$
\left\{\begin{array}{l}
P\left(\epsilon_{i}>t\right)=c p t^{-\alpha}\left\{1+b t^{-\beta}+o\left(t^{-\beta}\right)\right\} \\
P\left(\epsilon_{i}<-t\right)=c(1-p) t^{-\alpha}\left\{1+d t^{-\beta}+o\left(t^{-\beta}\right)\right\}
\end{array}\right.
$$

where $c>0, \alpha>0, p \in[0,1], b \neq 0, d \neq 0$ and $\beta>0$.

From Theorem 2.1 we have as $t \rightarrow \infty$

$$
\left\{\begin{array}{l}
P\left(Z_{i}>t\right)=c\left\{1+(1-\theta)^{\alpha}+|\theta|^{\alpha}\right\} t^{-\alpha}\left\{1+k_{1} A(t)+o(A(t))\right\} \\
P\left(W_{i}>t\right)=c\left\{1+(1+\theta)^{\alpha}+|\theta|^{\alpha}\right\} t^{-\alpha}\left\{1+k_{2} A(t)+o(A(t))\right\}
\end{array}\right.
$$

where $k_{i}=k_{i}(\alpha, \beta, \theta, c, b, d, p)(i=1,2)$ and

$$
A(t)= \begin{cases}t^{-\alpha} \vee t^{-\beta} & \text { if } \alpha<1 \\ \frac{\log t}{t} \vee t^{-\beta} & \text { if } \alpha=1 \\ t^{-\alpha} \vee t^{-\beta} & \text { if } 1<\alpha<2 \\ t^{-1} \vee t^{-\beta} & \text { if } \alpha \geq 2\end{cases}
$$

Note that it is possible, but tedious to give explicit expressions for $k_{1}$ and $k_{2}$. We omit the details.

Hence, similar to the prooof of Proposition 2.1 of Resnick and Starica (1997), we have

$$
\left\{\begin{array}{l}
\sqrt{k}\left(\hat{\alpha}_{Z}(k)-\alpha\right) \stackrel{d}{\rightarrow} N_{1}\left(\lambda_{1}, \alpha^{2}\left(1+2 \frac{1 \wedge(1-\theta)^{\alpha}+|\theta|^{\alpha}+(1-\theta)^{\alpha} \wedge|\theta|^{\alpha}}{1+(1-\theta)^{\alpha}+|\theta|^{\alpha}}\right)\right) \\
\sqrt{m}\left(\hat{\alpha}_{W}(m)-\alpha\right) \stackrel{d}{\rightarrow} N_{2}\left(\lambda_{2}, \alpha^{2}\left(1+2 \frac{1 \wedge(1+\theta)^{\alpha}+|\theta|^{\alpha}+(1+\theta)^{\alpha} \wedge|\theta|^{\alpha}}{1+(1+\theta)^{\alpha}+|\theta|^{\alpha}}\right)\right)
\end{array}\right.
$$


provided that as $n \rightarrow \infty$

$$
\left\{\begin{array}{l}
k \rightarrow \infty, \quad k / n \rightarrow 0 \\
m \rightarrow \infty, \quad m / n \rightarrow 0 \\
\sqrt{k} A_{1}(n / k) \rightarrow \lambda_{1} \in R, \quad \sqrt{m} A_{2}(n / m) \rightarrow \lambda_{2} \in R \\
\text { either } \quad \limsup \sup _{n \rightarrow \infty} n / k^{3 / 2}<\infty \text { or } \quad \liminf _{n \rightarrow \infty} n / k^{3 / 2}>0 \\
\text { either } \quad \limsup _{n \rightarrow \infty} n / m^{3 / 2}<\infty \text { or } \quad \liminf _{n \rightarrow \infty} n / m^{3 / 2}>0 .
\end{array}\right.
$$

Let $U(t)$ denote the inverse function of $\frac{1}{P\left(Z_{i}>t\right)}$. Note that

$$
\begin{aligned}
& \frac{k}{n} Z_{n, n-k}^{\hat{\alpha}_{Z}(k)}-c\left(1+(1-\theta)^{\alpha}+|\theta|^{\alpha}\right) \\
= & \left\{\frac{k}{n} Z_{n, n-k}^{\hat{\alpha}_{Z}(k)}-\frac{k}{n} Z_{n, n-k}^{\alpha}\right\}+\frac{k}{n} U^{\alpha}(n / k)\left\{\frac{Z_{n, n-k}^{\alpha}}{U^{\alpha}(n / k)}-1\right\} \\
& +\left\{\frac{k}{n} U^{\alpha}(n / k)-c\left(1+(1-\theta)^{\alpha}+|\theta|^{\alpha}\right)\right\} \\
= & \left\{\frac{k}{n} Z_{n, n-k}^{\alpha}\left(\hat{\alpha}_{Z}(k)-\alpha\right) \log Z_{n, n-k}\right\}\left(1+o_{p}(1)\right) \\
& +\frac{k}{n} U^{\alpha}(n / k)\left\{\frac{Z_{n, n-k}^{\alpha}}{U^{\alpha}(n / k)}-1\right\} \\
& +\left\{\frac{k}{n} U^{\alpha}(n / k)-c\left(1+(1-\theta)^{\alpha}+|\theta|^{\alpha}\right)\right\} .
\end{aligned}
$$

Using the fact that $Z_{n, n-k} / U(n / k)=1+O_{p}(1 / \sqrt{k})$, we have

$$
\begin{array}{ll} 
& \frac{\sqrt{k}}{\log (n / k)}\left\{\frac{k}{n} Z_{n, n-k}^{\hat{\alpha}_{Z}(k)}-c\left(1+(1-\theta)^{\alpha}+|\theta|^{\alpha}\right)\right\} \\
\stackrel{d}{\rightarrow} & N_{1}\left(\alpha \lambda_{1}, \alpha^{4}\left(1+2 \frac{1 \wedge(1-\theta)^{\alpha}+|\theta|^{\alpha}+(1-\theta)^{\alpha} \wedge|\theta|^{\alpha}}{1+(1-\theta)^{\alpha}+|\theta|^{\alpha}}\right)\right) .
\end{array}
$$

Similarly,

$$
\begin{aligned}
& \frac{\sqrt{m}}{\log (n / m)}\left\{\frac{m}{n} W_{n, n-m}^{\hat{\alpha}_{W}(m)}-c\left(1+(1+\theta)^{\alpha}+|\theta|^{\alpha}\right)\right\} \\
\stackrel{d}{\rightarrow} & N_{2}\left(\alpha \lambda_{2}, \alpha^{4}\left(1+2 \frac{1 \wedge(1+\theta)^{\alpha}+|\theta|^{\alpha}+(1+\theta)^{\alpha} \wedge|\theta|^{\alpha}}{1+(1+\theta)^{\alpha}+|\theta|^{\alpha}}\right)\right) .
\end{aligned}
$$

Note that the above two normal limits are dependent and the dependence structure is unknown. However, by requiring $k / m \rightarrow 0$ which implies $\lambda_{1}=0$, we have

$$
\frac{\sqrt{k}}{\log (n / k)}\left\{\frac{k}{m} Z_{n, n-k}^{\hat{\alpha}_{Z}(k)} W_{n, n-m}^{-\hat{\alpha}_{W}(m)}-f_{n}(\theta)\right\} \stackrel{d}{\rightarrow} N\left(0, \sigma^{2}\right)
$$

where

$$
\sigma^{2}=c^{2}\left(1+(1+\theta)^{\alpha}+|\theta|^{\alpha}\right)^{2} \alpha^{4}\left\{1+2 \frac{1 \wedge(1-\theta)^{\alpha}+|\theta|^{\alpha}+(1-\theta)^{\alpha} \wedge|\theta|^{\alpha}}{1+(1-\theta)^{\alpha}+|\theta|^{\alpha}}\right\} .
$$

Hence, it follows that the estimator $\hat{\theta}_{n}$ defined in (1.7) satisfies the following 
Theorem 4.1. Suppose (1.4), (4.1) and (4.4) hold and $k / m \rightarrow 0$ as $n \rightarrow \infty$. Then

$$
\frac{\sqrt{k}}{\log (n / k)}\left\{\hat{\theta}_{n}-\theta\right\} \stackrel{d}{\rightarrow} N\left(0, \sigma^{2}\left(\frac{d}{d \theta} f^{-}(\theta)\right)^{2}\right) .
$$

Remark 3. If we can find the dependence structure between the two normal limits $N_{1}$ and $N_{2}$, then we may be able to take $m=k$ and choose the sample fraction $k$ in an optimal way as in the tail index estimation and extreme tail probability estimation (see Drees and Kaufmann (1998) and Hall and Weissman (1997)). This will be a part of our future work.

\section{References}

[1] N. Bingham, C. Goldie and J. Teugels (1987). Regular Variation. Cambridge University Press, Cambridge, UK.

[2] P.J. Brockwell and R.A. Davis (1991). Time Series: Theory and Methods, 2nd ed. Springer, New York.

[3] T.E. Copeland and J.F. Weston (1983) Financial theory and corporate policy, Addison-Wesley, Reading.

[4] M.M. Dacorogna, U.A. Muller, O.V. Pictet and C.G. de Vries(1998). Extremal Forex Returns in Extremely Large Data Sets, submitted.

[5] J. Danielsson and C.G. de Vries (1997). Tail index and quantile estimation with very high frequency data, Journal of Empirical Finance, 4, 241-25\%.

[6] J. Danielsson and C.G. de Vries (1998). Value-at-Risk and extreme returns, Tinbergen Institute discussion paper TI98-017/2.

[7] S. Datta and W.P. McCormick (1998). Inference for the tail parameters of a linear process with heavy tail innovations. Ann. Inst. Statist. Math. 50, 337-359.

[8] R.A. Davis, M. Chen and W.T.M. Dunsmuir (1995). Inference for MA(1) processes with a root on or near the unit circle. Probability and Mathematical Statistics, 15, 227-242. 
[9] R.A. Davis and W.T.M. Dunsmuir (1996). Maximum likelihood estimation for MA(1) processes with a root on or near the unit circle. Econometric Theory, 12, 1-29.

[10] R.A. Davis and T. Mikosch (1998). Gaussian likelihood-based inference for non-invertible MA(1) processes with $S \alpha S$ noise. Stochastic Processes and their Applications, 77, 99-122.

[11] K. Dowd (1998). Beyond value at risk, the new science of risk management, Wiley, Chichester.

[12] H. Drees and E. Kaufmann (1998). Selecting the optimal sample fraction in univariate extreme value estimation. Stochastic Processes Appl. 75(2), 149-172.

[13] E.F. Fama and M.H. Miller (1972). The theory of finance , Dryden Press, Hinsdale.

[14] W. Feller (1971). An Introduction to Probability and Its Applications. Volume II, John Wiley \& Sons, Inc.

[15] J. Geluk, L. de Haan, S. Resnick and C. Starica (1997). Second order regular vatiation, convolution and the central limit theorem. Stochatistic Processes and their Applications, 9, 139-159.

[16] J. Geluk, L. Peng (1999). An adaptive optimal estimate of the tail index for MA(1) time series. Stat. Prob. Letters, to appear.

[17] L. de Haan and T. T. Pereira (1999). Estimating the index of a stable distribution. Stat. Prob. Letters, 41(1), 39-55.

[18] P. Hall and I. Weissman (1997). On the estimation of extreme tail probabilities. Ann. Statist. 25(3), 1311-1326.

[19] B.M. Hill (1975). A simple general approach to inference about the tail of a distribution. Ann. Statist. 3,1163-1174.

[20] P. Jorion 1997, Value-at-Risk, Irvin.

[21] K. Lii and M. Rosenblatt (1982). Deconvolution and estimation of transfer function phase and coefficients for nongaussian linear processes. The Annals of Statistics, 10(4), 1195-1208. 
[22] K. Lii and M. Rosenblatt (1992). An approximate maximum likelihood estimation for non-Gaussian non-minimum phase moving average processes. Journal of Multivariate Analysis, 43, 272-299.

[23] F.M. Longin (1997). From value-at-risk to stress testing:the extreme value approach, CERSSEC working paper 97-004.

[24] D.M. Mason (1982). Laws of large numbers for sums of extreme values. Ann. Prob. 10, 754-764.

[25] S. Resnick and C. Starica (1997). Asymptotic behavior of Hill's estimator for autoregressive data. Comm. Statist. Stochastic Models, 13(4), 703721. 